\title{
SIGNAL DETECTION FOR SPATIALLY MULTIPLEXED MIMO SYSTEM
}

\author{
Sanjeev Gogga ${ }^{1}$, Geeta Birkure ${ }^{2}$, Hameed Miyan ${ }^{3}$ \\ ${ }^{I}$ Assoc Prof, E\&CE Dept, BKIT Bhalki \\ ${ }^{2}$ M.Tech Scholar (DCN) BKIT, Bhalki \\ ${ }^{3}$ Prof. ECE Dept,BKIT Bhalki, India
}

\begin{abstract}
In this paper; we are studying techniques for signal detection in Multiple Input Multiple Output (MIMO) spatial multiplexing system. This paper includes techniques like Maximum likelihood (ML). The ML detection achieves the optimal performances with reduced complexity using BPSK modulation. The simulation result of ML decoder shows the Bit error rate (BER) reduces if signal to noise to ratio increases.
\end{abstract}

Keywords: MIMO, Spatial Multiplexing, Detection technique, Maximum likelihood detection, Equalization based detection technique.

\section{INTRODUCTION}

MIMO system can defined as point to point communication having multiple numbers of antennas at the transmitter and receiver side. By using multiple antennas at both sides clearly gives the performance enhancement over diversity systems. In recent research it is observed that MIMO can drastically increase the data rates of systems with no increase in the transmitting power and bandwidth of the system [1] [2]. Multiple inputs multiple output channels gives many advantages compared to Single Input Single Output (SISO) channels like array gain, the diversity, and the multiplexing gain. MIMO can yield significantly improve reliability of link and increase data rate without using additional bandwidth. The unique characteristic of MIMO channels is multiplexing gain. Spatial multiplexing is a multiplexing technique in MIMO to transmit separate and independent data signals is also called as streams, from each of the different transmitting antenna. Thus, spatial multiplexing is powerful technique which increases channel capacity at higher SNR values [3-5]. There are numerous detection techniques in MIMO wireless communication such as Maximum Likelihood detection (ML); Equalization based detection, sphere projection algorithm.

In this paper, we propose a ML detection technique using BPSK modulation. Results of simulation demonstrate significant improvement in performance and BER. The main advantage of the proposed technique is increasing the performance of received signal in order to maintaining the fixed and less computational complexities.

\section{SYSTEM MODEL}

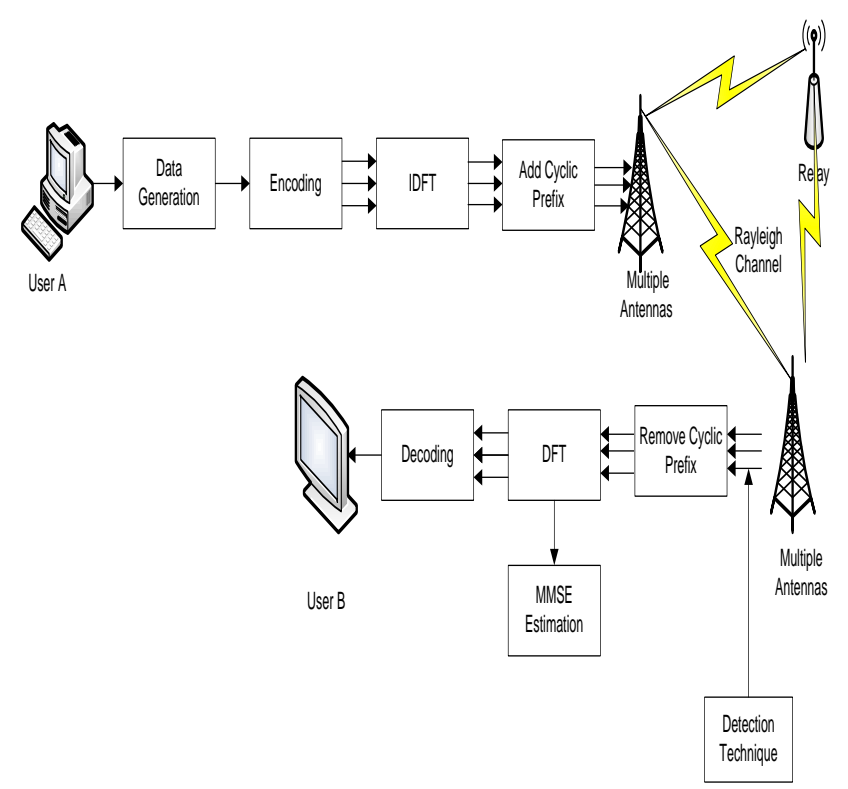

Fig 1: Block Diagram of MIMO Detection technique

Figure1 shows the block diagram MIMO detection technique. Detection is technique of extracting original signal from received signal. Maximum likelihood; Equalization based detection is used in proposed system. In MIMO high data rate is achieved by the mean of spatial multiplexing technique, in which Independent stream of information is transmitted in parallel over the different transmitting antenna [6]. Here, we consider fading channel in MIMO with $\mathrm{N}_{\mathrm{T}}$ antenna for transmitting, $\mathrm{N}_{\mathrm{R}}$ antennas at receiving and $\mathrm{N}_{\mathrm{R}} \geq \mathrm{N}_{\mathrm{T}}$. In MIMO spatial multiplexing system $n^{\text {th }}$ information symbol $b_{\mathrm{m}}$ is transmitted directly on $n^{\text {th }}$ transmit antenna. At a time instant this gives a baseband model with 


$$
\mathrm{v}=\mathrm{Hb}+\mathrm{c}
$$

The $\mathrm{N}_{\mathrm{T}} \times 1$ transmit vector $\mathrm{b}=\left(\mathrm{b}_{1} \mathrm{~b}_{2} \ldots \mathrm{B}_{\mathrm{NT}}\right)^{\mathrm{T}}$, The channel matrix $H$ is $N_{R} \times N_{T}$ the $N_{R} \times 1$ received vector $v=\left(v_{1} v_{2} \ldots\right.$. $\left.\mathrm{V}_{\mathrm{NT}}\right)^{\mathrm{T}}$, and $\mathrm{N}_{\mathrm{R}} \times 1$ noise vector $\mathrm{c}=\left(\mathrm{c}_{1} \mathrm{c}_{2} \ldots \mathrm{C}_{\mathrm{NR}}\right)^{\mathrm{T}}$. The $(\mathrm{n}, \mathrm{m})$ th entry of $\mathrm{H}, H_{\mathrm{n}, \mathrm{m}}=(\mathrm{H})_{\mathrm{n}, \mathrm{m}}$, is complex fading coefficient between the the transmitting and receiving antenna. The $b_{\mathrm{m}}$ is data symbol taken from a complex valued symbol $A$ and is considered to be mean zero and independent with unit variance. The $c_{m}$ is noise and it is considered to be symmetric in circularly with $\sigma_{w}{ }^{2}$ variance. At the receiver side, channel $\mathrm{H}$ is considered to be purely known [6].

The diversity in a MIMO SM system can be shown by the number of receive antenna $N_{\mathrm{R}}$. if $H_{\mathrm{n}, \mathrm{m}}$ channel coefficient is independent Maximum diversity is available, because each information data $b_{m}$ is transmitted over $N_{\mathrm{R}}$ independent scalar fading channels $H_{n, m}, n=1, \ldots, N_{\mathrm{R}}$ [6] . If larger is $N_{\mathrm{R}}$, smaller is the possibility that all of channels fade at a time and thus the data detection reliability can be increased. If $N_{R}$ available diversity, than the symbol error rate of Maximum likelihood detector technique decays like $S N R^{-M_{R}}$ in the high regime [7].

\subsection{Maximum Likelihood Detection}

Maximum likelihood is a perfect detection technique in terms of less error and it fully uses the available diversity. From system model (1) the maximum likelihood detection can be given by

$$
d_{M L}=\arg \min _{b \in D}\left\{\|v-H b\|^{2}\right\} .
$$

Here, $\mathcal{D}=\mathcal{A}^{N T}$ indicates the set of possible transmitted information vectors $b$. The cardinality of $|\mathcal{D}|=\left|\mathcal{A}^{N T}\right|$ and hence grows exponentially with $\mathrm{N}_{\mathrm{T}}$ [8] [9]. The maximum likelihood receiver tries to minimize $\hat{x}$ which minimizes,

$$
\begin{aligned}
& L=|d-H \hat{s}|^{2} \\
& {\left[\left(\begin{array}{l}
d_{1} \\
d_{2}
\end{array}\right)-\left(\begin{array}{ll}
h_{1,1} & h_{1,2} \\
h_{2,1} & h_{2,2}
\end{array}\right)\left(\begin{array}{l}
\hat{s}_{1} \\
\hat{s}_{2}
\end{array}\right)\right]^{2}}
\end{aligned}
$$

The simulated result include maximum likelihood detection in which main aim is to find the minimum among all the transmitted symbol based on the minimum chose that is estimate of transmitted data and it is repeated for more number of values of $E_{b} / N_{0}[10]$.

\subsection{Equalization Based Detection}

In equalization detection, an estimation of transmitted information $b$ is made as $d=G v$ with an "equalization matrix" G. The detected information vector is obtained as $\hat{b}=Q\{d\}$, where $\mathrm{Q}\{\cdot\}$ indicate the component wise according to the symbol $\mathcal{A}$ [6]. For the zero-forcing equalizer, the equalization matrix $\mathrm{G}$ is given by pseudo- inverse of $\mathrm{H}$, it is given by $\mathrm{G}=H^{\#}=\left(H^{H} \mathrm{H}\right)^{-1} \mathrm{H}^{\mathrm{H}}$. Thus, zero forcing equalization result is given by

$$
y_{Z F}=H^{\#} \mathrm{r}=\left(H^{H} H\right)^{-1} \mathrm{H}^{\mathrm{H}} \mathrm{r}=\mathrm{d}+\widetilde{w},
$$

The transmitted information vector $\mathrm{b}$ is lost by the the noise in channel $\tilde{c}=H^{H} \mathrm{c}$. This results the information caused by channel $\mathrm{H}$ is completely removed. The zero-forcing equalization received vector can be used as solution to the maximum likelihood detection problem, where the information set $\mathcal{D}$ underlying ML detection is relaxed to the convex set $\mathbb{C}^{M_{T}}[9]$.

$$
y_{Z F}=\arg \min _{y \in \mathbb{C}^{N T}}\left\{\|v-H d\|^{2}\right\}
$$

The noise effect in the ZF equalizer can be decreased by using the MMSE equalizer $\left(H^{H} H+\sigma^{2}{ }_{w} \mathrm{I}\right)^{-1} \mathrm{H}^{\mathrm{H}} \mathrm{r}$, and $\mathrm{G}$ minimizes the mean square error $\mathrm{E}\left\{\|\mathrm{G} v-b\|^{2}\right\}$, the MMSE equalization is given by

$y_{M M S E}=\left(H^{H} H+\sigma^{2}{ }_{w} \mathrm{I}\right)^{-1} \mathrm{H}^{\mathrm{H}} \mathrm{r}$

\section{RESULT ANOD DISCUSSION}

The Maximum likelihood detection tries to find the $\hat{x}$ in equation (2). The simulation ML decoder mainly include the finding the minimum among the four possible transmitted Symbols, based on the minimum chose the estimate of the transmitted symbol. This is repeated for no of values of Eb/N0 and plot the simulated result and theoretical results.

The figure 2 show performance of MIMO Maximum likelihood detection in terms of BER and SNR for $1 \times 2$ and $2 \times 2$ transmit diversity system. As per the graph BER decreases with increase in the SNR. At BER $\sim 10^{-3}$, the SNR values $\sim 11 \mathrm{~dB}$ for both the cases $1 \times 2$ and $2 \times 2$ transmit diversity and shows that the MIMO-ML detection shows same performance as those of $1 \times 2$ transmit diversity system.

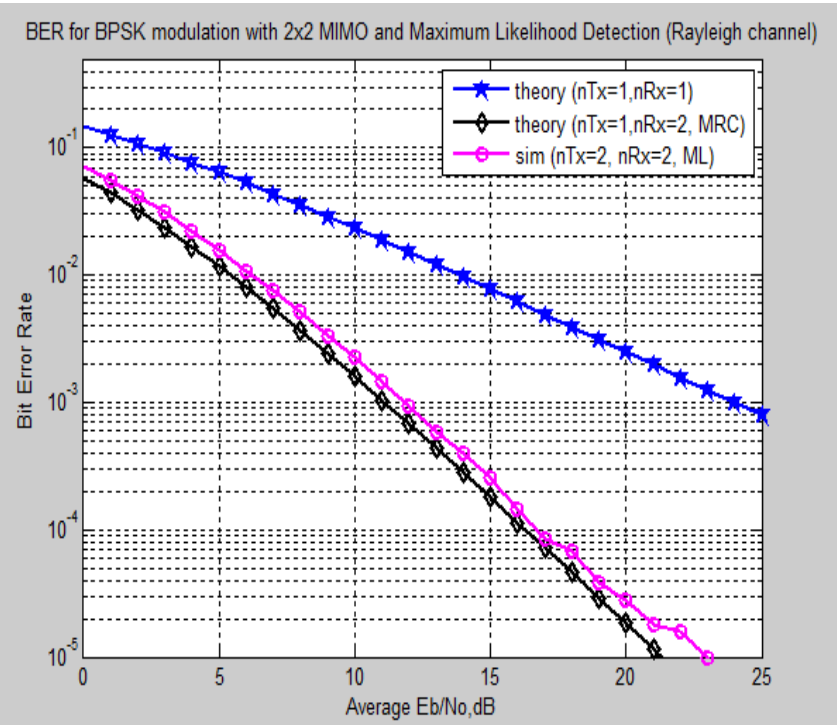

Fig 2: BER Performance with Maximum likelihood detection in MIMO 


\section{CONCLUSION}

The ML Detection is used to extract original signal from the received signal in MIMO. The Proposed ML detection gives the performance improvement compared to the conventional detection like equalization based and sphere decoding. The simulation result of $2 \times 2$ transmit diversity is close to the $1 \times 2$,It shows the bit error rate (BER) decreases as with the increase in the signal to noise ratio.

\section{REFERENCES}

[1]. Rajvirsinh. C Rana, Dr. Jag dish Rathod "Study and Analysis of Performance of Spatial Multiplexing equalizer for Transmit-Receive Diversity" International Journal (IJETTCS) April 2014.

[2]. B. Shim, J. W. Choi, and I. Kang, "Towards the Performance of ML and the complexity MMSE A hybrid approach," IEEE GLOBECOM'08.

[3]. D. Wubben, R. Bohnke, V. Kuhn, and K. D. Kammeyer, "Near maximum- likelihood detection of MIMO systems using MMSE-based lattice reduction," in Proc. IEEE ICC'06, Jun. 2006.

[4]. Thet Htun Khine, Kazuhiko Fukawa, and Hiroshi Suzuk "SuboptimalMaximumLikelihood Detection Using Gradient based Algorithm for MIMO Channels" IEEE 2006.

[5]. Anuj Vadhera1, Lavish Kansal "BER Analysis Of 3x3 MIMO Spatial Multiplexing under AWGN \& Rician Channels for Different Modulation Techniques" International Journal of Computer Science September 2013. [6]. Dominik Seethaler, Harold Artes, and Franz Hawatsch "Detection Technique for MIMO Spatial Multiplexing Systems" Elektrotechnik und Informationstechnik(e \& i) March 2005.

[7]. L. Zheng and D. Tse, "Diversity and Multiplexing: A fundamental tradeo_ in Multiple antenna channels" IEEE Trans. Inf. Theory May 2003.

[8]. L. Vandenberghe, and S. Boyd "Convex Optimization" Cambridge University Press, Dec. 2004.

[9]. Kon Max Wong, Zhi-Quan Luo, Wing Kin Ma, T. Davidson, and Pak-Chung Ching, "Quasi- maximumlikelihood Multiuser detection using semi-de_nite Relaxation with application to synchronous CDMA," IEEE Trans. Signal Processing, April 2002.

[10]. Abhishek Rawat, Sourabh Gaur "A Comparative Study of Equalization Techniques for MIMO Systems" Indian Journal of Research March 2013.

[11]. H. Artes, D. Seethaler, and F. Hlawatsch, "Efficient detection algorithms for MIMO Channels: A geometrical approach to Approximate ML detection," IEEE Trans. Signal Processing, Special Issue on MIMO Communications Systems, 2003.

[12]. B. M. Hochwald and S. ten Brink, "Achieving nearcapacity on a multiple-antenna channel," IEEE Trans. Inf. Theory, March2003. 